\title{
Surgical Management of Peritoneal Dialysis Peritonitis: The Impact of Peritoneal Sclerosis
}

\author{
Eli Mavor
}

Published online: 29 April 2009

(C) Société Internationale de Chirurgie 2009

Peritonitis is a major complication of peritoneal dialysis (PD) and remains the primary reason why patients switch from PD to hemodialysis [1]. Significant mortality rates (3.5-6.0\%) have been reported [2, 3]. Peritonitis associated with PD is most often due to contamination with pathogenic skin bacteria. Most cases of catheter-related peritonitis resolve with intraperitoneal instillation of antibiotics; for refractory peritonitis, when there is no response to appropriate intraperitoneal antibiotics the catheter should be removed. Still, in $10 \%$ to $15 \%$ of patients with primary PD peritonitis, these conservative measures fail. They then require laparotomy, lavage of the peritoneal cavity, and insertion of abdominal drains [4].

Another well known sequela of long-term PD is sclerosis of the peritoneum, which may be the common, usually innocuous, simple type or the rarer encapsulating type with its grave clinical outcome [5]. Up to now, the simple peritoneal sclerosis (SPS) form has been considered to have a low clinical impact, and it may be an incidental finding during abdominal surgery.

Yates et al. [6] demonstrated that the usually benign nature of SPS in PD patients may be a significant cause of morbidity if these patients develop refractory peritonitis requiring laparotomy. These authors examined the outcome of 62 PD patients who required laparotomy to treat their primary peritonitis from the perspective of the presence or absence of SPS. Their results confirmed the previously described association of SPS with longer duration PD. However, the major finding in this study was a significantly increased incidence of major complications following

E. Mavor $(\square)$

Department of Surgery A, Kaplan Medical Center, Rechovot, Israel

e-mail: mavored@netvision.net.il laparotomy in patients found to have SPS during the operation, in contrast to those without SPS. There was also an increased mortality rate for the SPS group, although it did not reach statistical significance.

These findings, demonstrating the possible adverse effect of SPS on the postoperative outcome in PD patients with peritonitis, may have important clinical implications. Given this observation and the increased likelihood of the presence of SPS with prolonged-duration PD, patients who are on long-standing PD should be treated more aggressively when they develop peritonitis, including early catheter removal and timely surgical intervention. Finally, chronic PD patients with confirmed SPS probably should be converted to hemodialysis to prevent the grave consequences of possible peritonitis.

\section{References}

1. Holley HL, Piraino BM (1990) Complications of peritoneal dialysis: diagnosis and management. Semin Dial 3:245

2. Perez Fontan M, Rodriguez-Carmona A, Garcia-Naveiro R et al (2005) Peritonitis related mortality in patients undergoing chronic peritoneal dialysis. Perit Dial Int 25:274-284

3. Mujais S (2006) Microbiology and outcomes of peritonitis in North America. Kidney Int Suppl (103):S55-S62

4. Troidle L, Finkelstein F (2006) Treatment and outcome of CPDassociated peritonitis. Ann Clin Microbiol Antimicrob 5:6

5. Garosi G, Di Paolo N (1999) Peritoneal sclerosis: an overview. Adv Perit Dial 15:185-192

6. Yates PJ, Kitchen JPA, Kaushik M et al (2009) Surgical management of peritoneal dialysis peritonitis: the impact of peritoneal sclerosis. World J Surg. doi: 10.1007/s00268-009-0027-1 